RAP CONFERENCE PROCEEDINGS, VOL. 5, PP. 55-58, 2020

ISSN 2737-9973 (ONLINE)| DOI: 10.37392/RAPPROC.2020.13

RAP-PROCEEDINGS.ORG

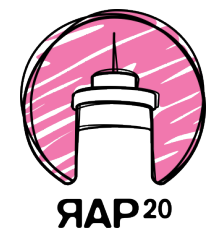

\title{
MEASUREMENT, EXPOSURE AND RISK ASSESSMENT OF OPTICAL RADIATION IN WORKING ENVIRONMENT
}

\author{
M. Ivanova ${ }^{1^{*}}$, M. Israel ${ }^{1,2}$, M. Stoinovska ${ }^{2}$ \\ ${ }^{1}$ National Center of Public Health and Analyses, Sofia, Bulgaria \\ ${ }^{2}$ Medical University - Pleven, Bulgaria
}

\begin{abstract}
The report presents results of the measurement, exposure and risk assessment of optical radiation sources in an industrial unit: electric welding, oxygene and plasma cutting machines. Measurements of the optical radiation parameters are performed over the entire optical range within the scope of Directive 2006/25/EC (transposed in Bulgarian legislation with Ordinance No 5 /2010). They are made at the level of the exposed eyes and skin of workers having activities or staying in the source area. The studied sources mainly emit in the ultraviolet (UV) and visible range of the optical spectrum, therefore the applicable exposure limit values (ELVs) correspond to the two ranges. Although the highest exposure to optical radiation is to the workers who directly handle the source, the exposure and risk assessment refers more to other workers indirectly involved in the activities with sources of optical radiation. The reason is that the first group of directly exposed workers is protected by personal protective equipment (PPE), so radiation does not reach them up to the maximal radiation levels. The exposure assessment results show an exceeding of the ELVs for the visible and UV range in the vicinity of the electric welding and plasma cutting machine and ELVS for the visible range for the oxygene. The risk assessment for workers has taken into account that the risk of exposure of the eye to visible light is high, but exposure to visible radiation is unlikely to occur due to the aversion to bright light and involuntary turning the head away from the source. This is not the case with exposure to UV radiation, which is invisible to the eye and there are no natural mechanisms for protection. So, high levels of exposure and risk to the cornea and the lens of the eye are possible. This means that the risk of exposure to visible optical radiation is high, but the probability of exposure is medium to low. In the ultraviolet range, the risk and the likelihood of exposure is high. There is a health risk to persons who are particularly sensitive to exposure to optical radiation as well. In addition, effects on the health and safety of workers are possible as a result of the interaction between optical radiation and photosensitising substances at the workplace or medications and/or food.
\end{abstract}

Keywords: Exposure assessment, exposure limit values, optical radiation, risk assessment, UV, visible

\section{INTRODUCTION}

There is no scope of human activity that is not connected to optical radiation exposure. In some of the cases, optical radiation is generated as a side product of the technological process, such as metalworking, welding, and in other cases, it is developed for purpose to achieve certain goals: lighting, medical therapy, disinfection and others. Regardless of what the origin of generated optical radiation exposure is, assessment is an essential part of physical factors of risk assessment in the working environment.

Notwithstanding the fact that Directive 2006/25/EC on the minimum health and safety requirements regarding the exposure of workers to risks arising from physical agents (artificial optical radiation) [1] has been transposed into national legislation as early as in 2010 with Ordinance No. 5 SG No.49/2010 [2], it is still not sufficiently implemented in practice for protecting workers from the impact of optical radiation in the working environment.
Optical radiation is often neglected as a physical factor of the working environment and the efforts are concentrated on lighting of the work place which is a "technological" element of the working environment, not necessarily connected with health but comfort and ability to perform work tasks.

The lack of competence and interest in this area results in missing of any control over the sources of optical radiation.

So, collecting a database of measurements and exposure assessment according to the requirements of the Directive 2006/25/EC is an important step towards establishing control over optical radiation in the working environment.

\section{AIM OF THE STUDY}

The aim of the study is to make exposure and risk assessment of optical radiation in workplaces and in zones around sources of optical radiation where the

*mihaela_1970@abv.bg 
personnel could pass along or perform activities not connected to optical radiation sources.

\section{MATERIALS AND METHODS}

\subsection{Sources of radiation}

The studied sources of optical radiation are plasma cutting, arc and oxygen welding machines, located in a large mechanical workshop. The considered facilities emit optical radiation as a by-product of their operation.

\subsection{Measurement equipment}

Measurements were conducted using the following equipment: Photo-Radiometer/Dosimeter HD2402, Delta Ohm, Italy with a Photometric sensor for visible radiation $(380 \div 780 \mathrm{~nm})$ - luxmeter; Radiometric sensor for UV radiation in the frequency band $(220 \div$ $400 \mathrm{~nm}$ ); Radiometric sensor for UVA radiation in the frequency band ( $315 \div 400 \mathrm{~nm}$ ); Radiometric sensor for blue light in the frequency band $400 \div 700 \mathrm{~nm}$; Radiometric sensor for IR radiation in the frequency band $700 \div 1300 \mathrm{~nm}$; Thermopile sensor for far IR $400 \div 2800 \mathrm{~nm}$ ).

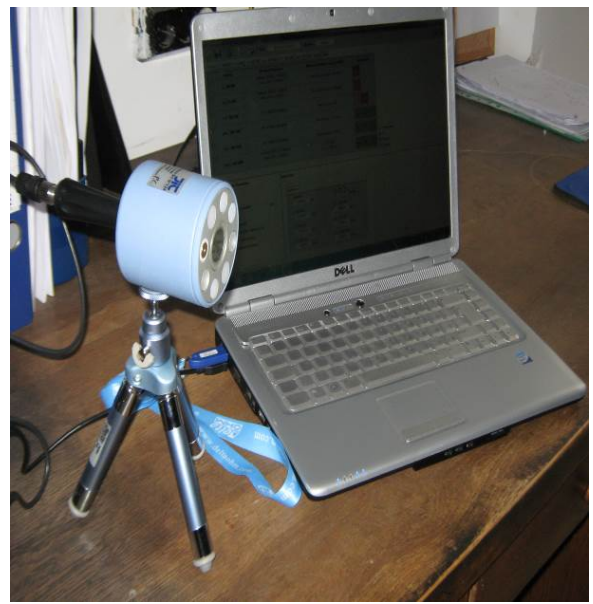

Figure 1. Photo-Radiometer/Dosimeter HD2402, Delta Ohm, Italy

\subsection{Measurement and assessment parameters:}

- Irradiance (power density) - E, W.m-2

- Radiance - L, W.m-2 ${ }^{-2} \mathrm{sr}^{-1}$;

- Radiant exposure - H, J.m².

\section{MEASUREMENT/ASSESSMENT METHOD}

The measurements of optical radiation parameters were performed on the basis of a Non-Binding Guide to the Artificial Optical Radiation Directive 2006/25/EC, EC [3], and EN62471:2008 [4].

The investigated sources mainly emit in the ultraviolet and visible range of the optical radiation spectrum, therefore the applicable exposure limit values refer to the two ranges. With regard to the visible range, the exposure limit values corresponding to the time duration of exposure and the angular subtense of the sources at assessed distances in working environment are applied.

The following considerations and approaches are used in the measurement and exposure assessment:

\subsection{Plasma Cutting}

The plasma cutting machine is located in a production hall in the workshop, and the control panel is located in a cabin where the workplace is situated.

Measurements to assess optical radiation are performed in the cabin at the level of the eyes of workers as the worst possible case of exposure. Additionally, for the purpose of exposure assessment of workers with different activities or those passing through the source area, measurements were made at two distances in the hall of the workshop on the level of possible exposed parts of the body: eyes and hands. Only hands are considered on exposure assessment of the skin except for the face because the rest of the body is covered by work clothes.

Geometrical factors used for the assessment in the visible range of the optical spectrum:

- Average source dimension of the source - for the average source dimension, the diameter of the electrode is considered $-\mathrm{d}=0.002 \mathrm{~m}$; the source is considered to be homogeneous;

- Angle between the perpendicular to the source surface and the line-of-sight is $\theta=20^{\circ}$ for a working position in the cabin (eye level for a seated working posture); $\theta=45^{\circ}$ outside the cabin at the eye level of the residents in the source area;

- Distances to the source - $\mathrm{L}=2.36 \mathrm{~m}$ - along the outline on the floor where the access of workers not connected with the process is not allowed; $\mathrm{L}=3.7 \mathrm{~m}$ in the middle of the main path in the workshop.

The exposure assessment was performed for the angular subtense of the source $1.12 \mathrm{mrad}$ in the cabin and from $0.76 \mathrm{mrad}$ to $1.19 \mathrm{mrad}$ at the various measuring points in the workshop hall.

\subsection{Arc welding}

Electrical arc welding is done directly in the workshop hall, without protective enclosure around the source. During the measurements, typical electrodes are used, with current ranging from 130 to $180 \mathrm{~A}$.

Measurements are performed at different distances in the closest possible access points to assess the exposure of workers or persons in the source zone, at eye/face and hands level.

Geometrical factors used for assessment in the visible range of the optical spectrum:

- Average source dimension - the diameter of the electrode $-\mathrm{d}=0.004 \mathrm{~m}$; the source is considered to be homogeneous;

- Angle between the perpendicular to the source surface and the line of sight $\theta=60^{\circ}$ during the welding of a piece located on the ground;

- Distances to the source $-\mathrm{L}=1.5 \mathrm{~m} ; \mathrm{L}=3.0 \mathrm{~m}$ - to the center of the main path in the workshop hall.

The exposure assessment was performed for the angular subtense of the source from $2.67 \mathrm{mrad}$ to $5.33 \mathrm{mrad}$ at different measurement points in the workshop. 


\subsection{Welding with oxygen}

The work tasks with oxygen are done directly in the workshop, without protective enclosure around the source. During the measurements, the workpiece is located at a height of $\mathrm{h}=0.70 \mathrm{~m}$ from the floor.

Measurements are performed at different distances in the closest possible access points to assess the exposure of workers or persons in the source zone, at eye/face and hands level.

Geometrical factors used for assessment in the visible range of the optical spectrum:

- Average source dimension - the diameter of the electrode is considered $-\mathrm{d}=0.001 \mathrm{~m}$ (nozzle № 3 ); the source is considered to be homogeneous;

- Angle between the perpendicular to the source surface and the line of sight is $\theta=30^{\circ}$ during the welding of a piece located on the ground;

- Distance to the source $-\mathrm{L}=1.0 \mathrm{~m} ; \mathrm{L}=2.0 \mathrm{~m}$; $\mathrm{L}=3.0 \mathrm{~m} ; \mathrm{L}=4.0 \mathrm{~m}$ to the center of the main path in the workshop.

The exposure assessment was performed for the angular subtense of the source from $0.29 \mathrm{mrad}$ to $0.57 \mathrm{mrad}$ at different measurement points in the workshop.

The results of the eye level measurements are compared with the eye and skin limit values of the workers corresponding to the spectral range. In the case of workers' hands, only skin exposure limit values have been applied, taking into account the possible exposure of workers passing through the workshop or performing activities which are not directly related to the investigated process.

When carrying out activities directly with the three sources studied, the workers are using the personal protective equipment (PPE).

\section{RESULTS}

Measurements are made to evaluate the emitted optical radiation from considered sources across the optical spectrum. The results are evaluated against all applicable indices in terms of the emission spectrum, source dimension, as required by the Directive $2006 / 25 /$ EC [1]. Here, only the results that are of interest concerning the health risk of workers at exposure to optical radiation are presented.

\subsection{Plasma cutting}

The results of the measurement and exposure assessment indicate that, despite the separation of the workplace from the source by placing the command board in a cabin, there are still values of radiance above the exposure limit values for the visible range - blue light risk for an 8-hour working day. The material from which the protective cabin is built prevents the penetration of ultraviolet radiation from the source. This is not the case, however, for the sites surveyed in the source area in the production hall of the workshop. At these points, the exposure limit (for ultraviolet radiation) is exceeded.

The results from measurements inside the cabin with the command board of plasma cutting machine are presented in Figure 2.

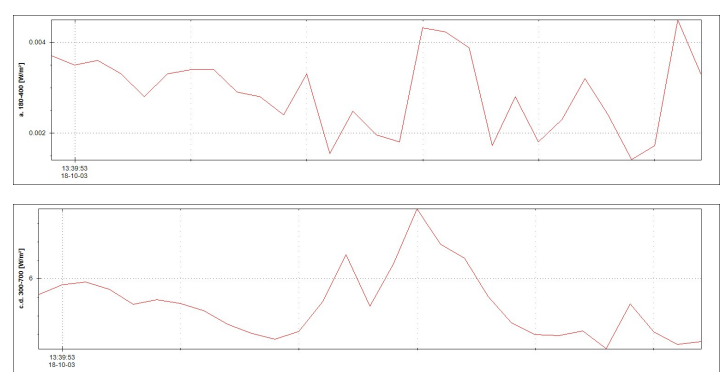

Figure 2. Results from measurements inside the cabin with the command board of plasma cutting machine

The results from measurements outside the cabin at a distance $\mathrm{L}=2.36 \mathrm{~m}$ from the plasma cutting machine at eye level are presented in Figure 3.
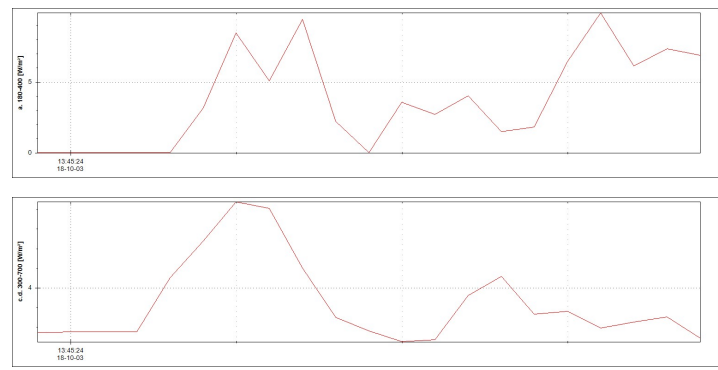

Figure 3. Results from measurements outside the cabin at a distance $\mathrm{L}=2.36 \mathrm{~m}$ from the plasma cutting machine at eye level

5.2. Arc welding

The measured radiance values at studied places in the source area in the workshop hall exceed the exposure limit values for an 8-hour working day for ultraviolet radiation and for visible radiation (blue light).

The results from measurements at a distance $\mathrm{L}=3.00 \mathrm{~m}$ from the arc welding machine at eye level are presented in Figure 4.

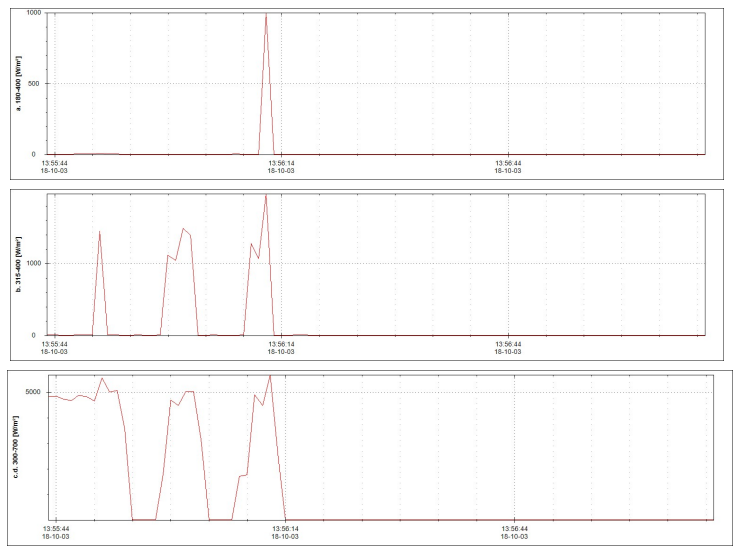

Figure 4. Results from measurements at a distance $\mathrm{L}=3.00 \mathrm{~m}$ from the arc welding machine at eye level

\subsection{Welding with oxygen}

At the studied locations in the source area in the workshop hall, the measured values of radiance exceed the limit values for visible radiation. 
The results from measurements at a distance $\mathrm{L}=1.00 \mathrm{~m}$ from the arc welding machine at eye level are presented in Figure 5 .

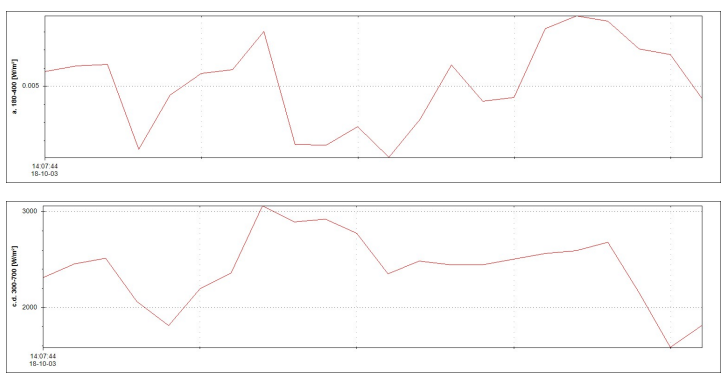

Figure 5. Results from measurements at a distance $\mathrm{L}=1.00 \mathrm{~m}$ from the arc welding machine at eye level

\section{DISCUSSION}

The risk assessment should take into account that since the sources of optical radiation are very bright, it can be assumed that the reaction of aversion to bright light, which is a universal reflex in humans, will limit episodes of exposure to $0.25 \mathrm{~s}$.

Taking into account the actual exposure time of the eyes with visible radiation, the time spent away from the source can be excluded. This means that the risk of irradiation with visible optical radiation is high, but the probability of exposure is medium / low.

Such assumptions are not possible with respect to exposure to ultraviolet radiation. In this case, the risk of exposure is high and the likelihood of exposure is high.

For this reason, the permissible exposure time determined on the basis of indices a. and b. (ultraviolet radiation) and noted in the table shall be observed in all cases.

Although the highest exposure to optical radiation refers to a worker who directly works with the studied source, this risk assessment is more relevant to other workers indirectly involved in the plasma cutting, welding, or oxygen welding. The reason for this is that the worker handling the source is protected by PPE - a face shield with a filter and protective clothing, and the optical radiation does not reach his body - eyes and skin to the levels commented above.

Data on measurements show that persons who are particularly sensitive to exposure to optical radiation are at particular health risk. Effects on the health of workers are possible as a result of the interaction between optical radiation and photo-sensitizing substances at the workplace or from medication and food.

\section{CONCLUSION}

The study covers workplaces with plasma cutting machines, arc and oxygen welding machines as sources of optical radiation. The results of performed measurements, exposure and risk assessment indicate a possible health risk from exposure to optical radiation not only for workers directly involved in activities with emitting equipment but also to the workers carrying out other activities or passing through in the radiation zone in the workshop.

The established results show the need for controlling the sources of optical radiation in the working environment and taking adequate protective measures. Protective measures should cover all workers staying on the premises under consideration. The results are used to propose protection measures that correspond to the risk level and the specificity and location of activities performed in the production workshop.

The obtained data could be used as examples of exposure and risk assessment and as good practice in the training of control bodies and measurement laboratories.

\section{REFERENCES}

1. The European Parliament and the Council of European Union. (Apr. 5, 2006). Directive 2006/25/EC on the minimum health and safety requirements regarding the exposure of workers to risks arising from physical agents (artificial optical radiation) (19th individual Directive within the meaning of article 16(1) of Directive 89/391/EEC).

Retrieved from:

https://eur-

lex.europa.eu/LexUriServ/LexUriServ.do?uri=OJ:L:20 06:114:0038:0059:en:PDF

Retrieved on: Jan. 15, 2020

2. Министерство на труда и социалната политика и Министерство на здравеопазването на България. (Июнь 11, 2010). Бр. 5. Наредба за минималните изисквания за осигуряване на здравето и безопасността на работещите при рискове, свързани с експозиция на изкуствени оптични льчения.

(Ministry of labor and Social Policy and Ministry of Health of Bulgaria. (Jun. 11, 2010). No. 5. Ordinance on the minimum health and safety requirements regarding the exposure of workers to risks arising from exposure to artificial optical radiation).

Retrieved from:

https://www.lex.bg/laws/ldoc/2135686489

Retrieved on: Jan. 10, 2020

3. A Non-Binding Guide to the Artificial Optical Radiation Directive 2006/25/EC, European Commission, Brussels, Belgium.

Retrieved from:

https://www.gla.ac.uk/media/Media 164337 smxx.pd $\underline{f}$

Retrieved on: Jan. 20, 2020

4. Photobiological safety of lamps and lamp systems, EN 62471, Sep. 12, 2008.

Retrieved from:

http://tbt.testrust.com/image/zt/123/100123_2.pdf Retrieved on: Aug. 15, 2019

5. D. H. Sliney, M. L. Wolbarsht, Safety with Lasers and Other Optical Sources, New York (NY), USA: Plenum, 1980.

Retrieved from: http://library.lol/main/9342391CoooB199172E887B73 095AF6F

Retrieved on: Feb. 27, 2020 